\title{
Utilização de técnicas de regressão logística na avaliação de vazões máximas do Rio Doce, no Município de Colatina, ES, Brasil
}

\author{
Using logistic regression techniques to evaluate of maximum flow rates of the Rio \\ Doce, city of Colatina, ES, Brazil
}

\author{
Wanderson de Paula Pinto*1, Gemael Barbosa Lima², Edson Zambon Monte ${ }^{3}$ e Claudinei \\ Antonio Montebeller 4 \\ ${ }^{1}$ Departamento de Ciências Ambientais, Faculdade da Região Serrana - FARESE, Santa Maria de Jetibá, ES, Brasil. \\ ${ }^{2}$ Departamento de Ciências Ambientais, Faculdade da Região Serrana - FARESE, Santa Maria de Jetibá, ES, Brasil. \\ ${ }^{3}$ Departamento de Economia, Universidade Federal do Espírito Santo - UFES, Vitória, ES, Brasil. \\ ${ }^{4}$ Instituto Capixaba de Pesquisa, Assistência Técnica e Extensão Rural - INCAPER e Centro Universitário do Espírito Santo - \\ UNESC, Colatina, ES, Brasil.
}

\begin{abstract}
Resumo
Esta pesquisa teve por objetivo avaliar os impactos das precipitações máximas na bacia hidrográfica do rio Doce na probabilidade de ocorrência de vazões de alerta de inundação no Município de Colatina, ES, por meio do modelo Logit. Para isso, consideraram-se as estações pluviométricas das localidades de Aimorés, Tumiritinga, Conselheiro Pena e Resplendor, no estado de Minas Gerais, e Baixo Guandu, Itaguaçu, Itarana e Colatina, no Espírito Santo, além da estação fluviométrica da cidade de Colatina, durante o período de 01/01/1986 a 31/12/2014. Os resultados do modelo de regressão logística demonstraram que existem impactos significativos das precipitações máximas dos municípios de Tumiritinga, Aimorés, Baixo Guandu e Colatina na probabilidade de ocorrência de episódios de vazão de alerta de inundação no município de Colatina, ES. Além disso, observou-se que nos períodos com maiores volumes de chuva (primavera e verão), a chance de ocorrência de um evento de vazão de alerta em Colatina aumentou significantemente. Por fim, conclui-se que os resultados desse artigo podem subsidiar ações direcionadas ao controle de enchentes.

Palavras-chave: Vazão de Alerta, Rio Doce, Logit, Bacia Hidrográfica, Enchente.
\end{abstract}

\section{Abstract}

This research aimed to evaluate the impacts of maximum rainfall in watershed of the Rio Doce in the probability of flooding alert flow in the municipality of Colatina, ES, Brazil, using Logit model. To this, it were considered the rainfall stations of the locations of Aimorés, Tumiritinga, Conselheiro Pena and Resplendor in the Minas Gerais state and Baixo Guandu, Itaguaçu, Itarana and Colatina in the state of Espirito Santo, beyond fluviometric station in Colatina municipality, during the period from 01/01/1986 to 31/12/2014. The results of the logistic regression model shown significant impacts of the maximums rainfall in Tumiritinga, Aimorés, Baixo Guandu and Colatina in the probability of occurrence of episodes of flood warning in Colatina, ES. Moreover, it was observed that in periods with higher volumes of rain (spring and summer), the chance of occurrence of a warning flow event in Colatina increased significantly. Lastly, it is hoped that this article can support actions aimed at flood control.

Keywords: Alert flooding flow, Rio Doce, Logit, watershed, Flooding. 


\section{Introdução}

Dentre os fatores que tem conduzido a depleção da qualidade/quantidade dos recursos hídricos brasileiros está o significativo crescimento demográfico. De acordo com Brito e Pinho (2012), o censo de 1970 registrava pela primeira vez que, durante os anos sessenta, a população urbana tinha superado a rural, no Brasil. Ainda, segundo o mesmo autor, na segunda metade do século passado, a população urbana passou de 19 milhões para 138 milhões, com uma taxa de crescimento médio anual de $4,1 \%$.

Todos os efeitos desse processo se fazem sentir no aparelhamento urbano relativo a recursos hídricos, a saber: abastecimento de água, transporte e tratamento de esgotos cloacais e drenagem urbana. Todavia, dentre os efeitos supracitados, ressalta-se a drenagem urbana, uma vez que as enchentes urbanas contribuem vertiginosamente para os impactos negativos sobre a sociedade (Tucci et al., 2015).

Segundo Mota (2008), à medida que a cidade se urbaniza, em geral, ocorre o aumento das vazões máximas em até sete vezes, devido ao aumento da capacidade de escoamento através de condutos e canais e da impermeabilização das superfícies. Nesse contexto, o conhecimento das vazões máximas de uma determinada localidade é necessário para a previsão de enchentes, no projeto de obras hidráulicas de obras de drenagem urbana, diques, extravasores, bem como o controle a atenuação das cheias (Tucci, 2012).

De acordo com o Rodriguez et al. (2009), o Brasil é um dos países mais afetados por inundações, causando grande número de vítimas e prejuízos econômicos. Em 2011 o Brasil registrou cerca de 3,7 milhões de vítimas durante o ano, sendo, predominantemente, vítimas de eventos meteorológicos. No ano de 2013, o número de pessoas mortas por inundações em todo mundo foi o maior registrado na década. As mortes devido à enchentes representam $45,4 \%$ das mortes por desastres naturais no mundo (Guha-Spir et al., 2014).

A necessidade de adotar medidas direcionadas a prevenir e mitigar os danos causados pelas recorrentes inundações nas bacias hidrográficas, tem conduzido o emprego de ferramentas estatísticas no tratamento de dados hidrológicos. Meller et al. (2012) descreveram um método empírico de assimilação de dados com a modelagem hidrológica distribuída, com o intuito de prever as cheias do rio Piracicaba. Os resultados apontaram que o método de assimilação tem impactos positivos nos resultados das previsões.

Hartmann et al. (2011) estimaram a precipitação pluvial máxima esperada, em Presidente Prudente, SP, para diferentes níveis de probabilidade, bem como verificaram o grau de ajuste dos dados ao modelo Gumbel, com as estimativas dos parâmetros obtidas pelo método de máxima verossimilhança. De acordo com os resultados apresentados, houve um bom ajuste da distribuição Gumbel para os dados da precipitação máxima mensal para a região de estudo. Os autores concluíram que as estimativas de precipitação obtidas pelo método de máxima verossimilhança são consistentes, conseguindo reproduzir com bastante fidelidade o regime de chuvas da região de Presidente Prudente.

Macêdo et al. (2013) avaliaram aspectos que influenciam o comportamento hidrológico da bacia hidrográfica do Riozinho do Rôla, por meio do uso de instrumentos de análise descritiva. Os autores verificaram que a distribuição da precipitação pluviométrica da bacia do Riozinho do Rôla é flutuante, o que influencia diretamente na dinâmica fluvial da área de drenagem da bacia. Também observaram picos de cheia no inverno, chegando a $200 \mathrm{~mm} / \mathrm{mês,}$ e estiagem no verão, com $9 \mathrm{~mm} / \mathrm{mês}$. Associado à precipitação, a vazão atinge picos que atingem $1.276,9 \mathrm{~m}^{3} / \mathrm{s}$ em período de cheia e 4,1 $\mathrm{m}^{3} / \mathrm{s}$ em períodos com menor pluviosidade. No mais, segundo Macêdo et al. (2013), esse comportamento está fundamentalmente relacionado aos aspectos naturais e sociais como: ocorrência de rede hidrográfica com características de cabeceiras associado a um regime hidrológico marcado pela alta sazonalidade, baixa permeabilidade de seus solos e intensificação do desmatamento na região, em sua maioria, com pecuária extensiva.

Pinto et al. (2015) realizaram estudo cujo objetivo foi analisar, comparativamente, modelos de séries temporais, bem como realizar previsões das vazões médias mensais para o rio Doce, em Colatina, ES, utilizando a metodologia de séries temporais proposta por Box e Jenkins (1970). Os autores compararam modelos de séries temporais que consideram ou não a presença da sazonalidade no conjunto de observações. Baseando-se em indicadores de desempenho de modelagem, verificaram que o modelo que considera a informação de sazonalidade (SARIMA) fornece previsões mais precisas. Após a etapa de validação do modelo foram feitas previsões para os anos de 2014 e 2015. De maneira geral, os resultados obtidos com a modelagem dos dados de vazão média mensal com o modelo SARIMA, tanto no ajuste quanto no estudo de previsão, foram considerados acurados.

Reis et al. (2016) realizaram um estudo no estado de Minas Gerais, cujo objetivo foi ajustar e validar a utilização de modelos de regressão polinomial para prever o crescimento do nível do rio Sapucaí em uma seção de interesse, a partir do nível observado em um ponto a montante, utilizando apenas registros de níveis fluviométricos, tendo 
em vista compor um sistema de monitoramento e alerta de inundações em tempo real para o município de Itajubá, MG. Após ajuste e calibração do modelo matemático, o modelo foi implementado na plataforma TerraMA, que resultou em um sistema de monitoramento capaz de produzir alertas consistentes com quatro horas de antecedência, apresentando alta correlação entre os dados observados e a série predita. Os autores concluíram que a utilização de modelos matemáticos para previsão de inundações, em substituição aos tradicionais modelos hidrológicos, mostrou-se eficaz. Conforme os resultados apontados no trabalho, esse sistema de alerta pode auxiliar a tomada de decisões, gerando uma estimativa confiável de crescimento de nível do rio, com objetivo de reduzir a perda de vidas e os danos materiais.

No que se refere à metodologia de estudo estatística proposta nesta pesquisa, destaca-se que, o modelo Logit é uma ferramenta estatística que permite o ajuste de um conjunto de variáveis independentes a uma variável dependente dicotômica, ou seja, é possível estimar probabilidades de ocorrências em variáveis dependentes do tipo binário. O modelo Logit aplicado na modelagem de dados ambientais tem sido utilizado por Kuchenho e Thamerus (1996), Silva et al. (2011), Mendes e Vega (2011), entre outros autores. Adicionalmente, não se verifica na literatura específica estudos para predizer a probabilidade de ocorrência de picos de vazões máximas por meio da utilização do modelo Logit.

Dessa forma, este trabalho objetivou avaliar os impactos das precipitações máximas na bacia hidrográfica do rio Doce na probabilidade de ocorrência de episódios de vazão de alerta de inundação, ou seja, vazões maiores ou iguais a $4131 \mathrm{~m}^{3} / \mathrm{s}$, no município de Colatina, ES, Brasil, no período de 01/01/1986 a 31/12/2014, por meio do modelo Logit. É importante dizer que a vazão de referência foi obtida junto ao CPRM - Serviço Geológico do Brasil (2015).

Vale ressaltar que, segundo Dallapicola e Coelho (2015), a área urbana do município de Colatina compreende um total de sessenta e dois bairros, representando uma área de 33,29 $\mathrm{km}^{2}$. Dezesseis bairros estão parcialmente localizados junto a planície de inundação da calha principal do rio Doce, sendo nove na margem esquerda e sete na margem direita do rio, representando uma área de $15,06 \mathrm{~km}^{2}$ em relação totalidade da área urbana do município.

\section{Materiais e métodos}

\section{1 Área de estudo}

A Bacia hidrográfica do rio Doce é uma importante bacia situada na região Sudeste, que por sua vez integra a região hidrográfica do Atlântico Sudeste, entre os paralelos $17^{\circ} 45^{\prime}$ e $21^{\circ} 15^{\prime} \mathrm{S}$ e os meridianos $39^{\circ} 30^{\prime}$ e $43^{\circ} 45^{\prime}$ W. As nascentes do rio Doce localizam-se nas serras da Mantiqueira e do Espinhaço no estado de Minas Gerais e tem sua foz no oceano Atlântico, em Regência no Espírito Santo (C.B.H. Doce, 2014).

Conforme apresentado em Pinto et al. (2015), a bacia hidrográfica do rio Doce (Figura 1) apresenta área aproximada de $83.400 \mathrm{~km}^{2}$, dos quais $86 \%$ pertencem ao Estado de Minas Gerais e o restante ao Estado do Espírito Santo. Abrange, total ou parcialmente, áreas de 228 municípios, sendo 202 em Minas Gerais e 26 no Espírito Santo. O rio Doce, com uma extensão de $853 \mathrm{~km}$, tem como formadores os rios Piranga e Carmo, cujas nascentes estão situadas nas encostas das serras da Mantiqueira e Espinhaço, onde as altitudes atingem cerca de $1.200 \mathrm{~m}$ e deságua no mar em Regência no município de Linhares, ES. Seus principais afluentes são: Santo Antônio, Suaçuí Grande, Casca, Matipó, Caratinga-Cuieté e Manhuaçu, em Minas Gerais e Pancas, São José e Guandu no Espírito Santo.

De acordo com a classificação de Köppen (Koppen, 1900), identificam-se três tipos climáticos na bacia, a saber: o clima tropical de altitude com chuvas de verão e verões frescos, presente nas vertentes das serras da Mantiqueira e do Espinhaço e nas nascentes do rio Doce; clima tropical de altitude com chuvas de verão e verões quentes, presentes nas nascentes de seus afluentes; e clima quente com chuvas de verão, presentes nos trechos médio e baixo do rio Doce e de seus afluentes. A precipitação média anual na bacia varia de $1.500 \mathrm{~mm}$, nas nascentes localizadas nas serras da Mantiqueira e do Espinhaço, a 900 mm, na região da cidade de Aimorés, MG, voltando a crescer em direção ao litoral (C.B.H. Doce, 2014).

A bacia hidrográfica do rio Doce é originalmente coberta por Mata Atlântica. A intensa devastação restringiu o revestimento florístico originário basicamente à área do Parque Estadual do rio Doce. As demais matas correspondem a uma vegetação que sofreu influência antrópica intensa, constituindo-se em vegetação secundária. Estima-se que menos de $7 \%$ da área possui hoje cobertura vegetal. Dessas, menos de $1 \%$ encontra-se em estágio primário. Na zona rural encontram-se vastas áreas em estado avançado de desertificação, lagoas eutrofizadas, nascentes desprotegidas e processos erosivos. Da cobertura vegetal original, mais de 90\% foi extinta (C.B.H. Doce, 2014).

A economia está baseada principalmente nas seguintes atividades: (I) agricultura, com destaque para a pecuária de leite e corte, suinocultura, café, cana-de-açúcar, hortifrutigranjeiros e cacau; (II) indústria: siderurgia, metalurgia, 
mecânica, química, alimentícia, álcool, têxtil, curtume, papel e celulose; e (III) mineração: ferro, ouro, bauxita, manganês, rochas calcáreas e pedras preciosas; e (IV) geração de energia elétrica com uma capacidade de geração de cerca de 4.055 MW (Pinto et al., 2015).

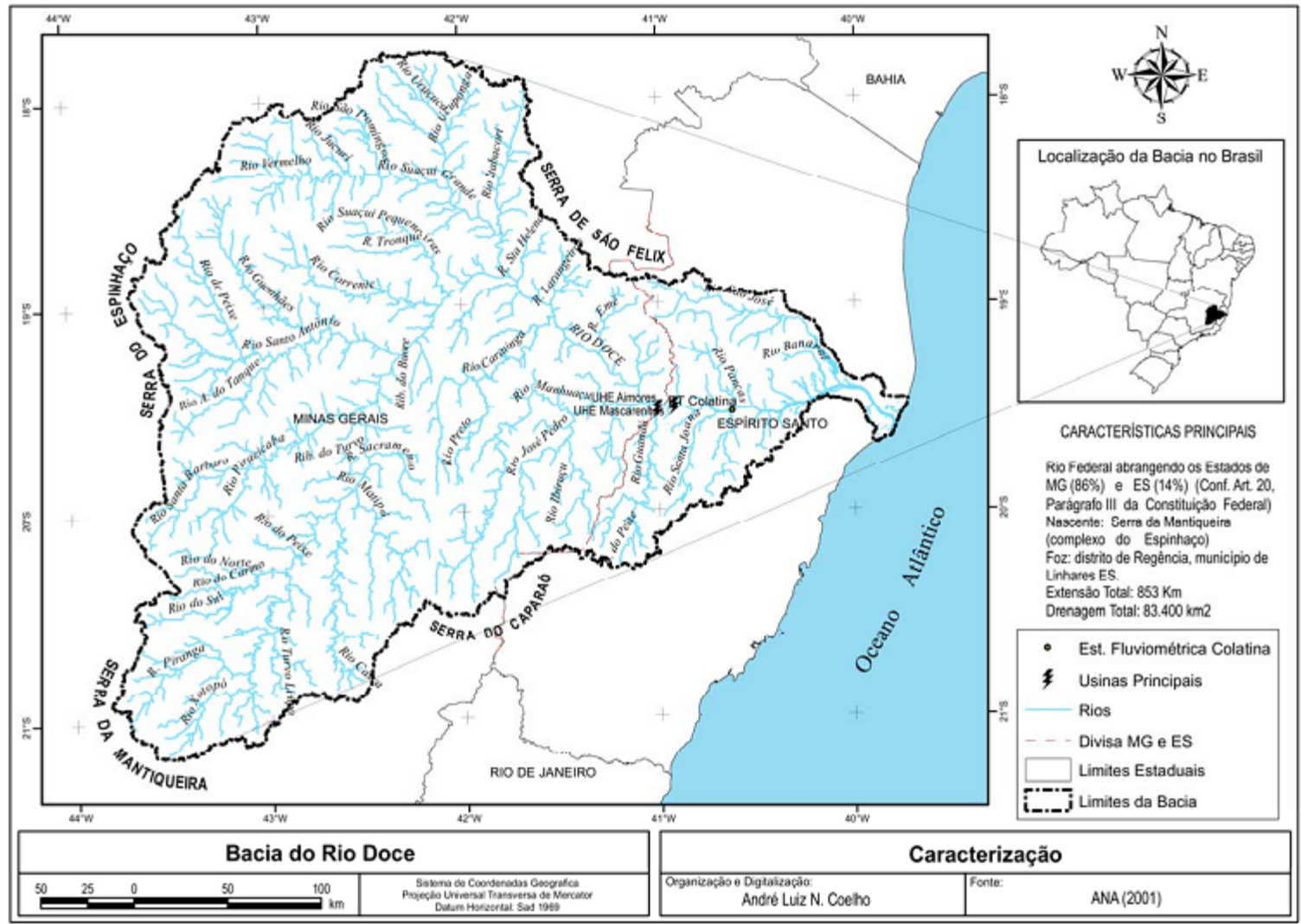

Figura 1: Localização da bacia hidrográfica do rio Doce e área de drenagem.

Fonte: Coelho (2007).

\subsection{Dados}

As séries históricas de precipitações máximas mensais e vazões máximas mensais utilizadas neste estudo foram obtidas junto ao Sistema de Informações Hidrológicas (HIDROWEB), mantido pela Agência Nacional de Água (ANA), disponível no site http://hidroweb.ana.gov.br/. As estações de monitoramento pluviométrico selecionadas, bem como o código e coordenadas geográficas estão apresentadas na Tabela 1.

Algumas estações de monitoramento operam desde o ano de 1939, porém como algumas só iniciaram a operação em 1980, para a utilização nesta pesquisa, utilizaram-se dados de vazões e precipitações máximas mensais do período de 01/01/1986 a 31/12/2014, resultando assim em séries históricas com dados de 29 anos. Tais séries apresentam dados faltantes, uma vez que podem acontecer falhas nos equipamentos de medição. No entanto, a imputação dessas obervações faltantes foi baseada na metodologia estudada por Junger e Leon (2015).

\subsection{Modelo Logit}

Para verificar a influência das precipitações aferidas nas estações pluviométricas da bacia hidrográfica do rio Doce na probabilidade de ocorrência de picos de vazões de alerta de inundação, foi utilizado o modelo Logit (Gujarati e Porter, 2008), que admite valores discretos, zero e um (variável binária), para a variável dependente. Foram considerados dados pluviométricos dos seguintes municípios da Bacia do estado de Minas Gerais: Tumiritinga (TUMIR), Conselheiro Pena (CPENA), Resplendor (RESPL) e Aimorés (AIMOR) e, do estado do Espírito Santo: Itarana (ITAR), Itaguaçu (ITAGUA), Baixo Guandu (BGUAD) e Colatina (COLAT). No mais, como a regressão logística considera a variável dependente como dicotômica, a série temporal de vazões máximas de Colatina foi 
Tabela 1: Coordenadas geográficas das estações pluviométricas da ANA cujos dados foram utilizados neste estudo

\begin{tabular}{lllc}
\hline Código & \multirow{2}{*}{ Estação } & \multicolumn{2}{c}{ Coordenadas } \\
\cline { 3 - 4 } & & Latitude & Longitude \\
\hline 1941010 & Aimorés & $-19^{\circ} 29^{\prime} 33^{\prime \prime}$ & $-41^{\circ} 09^{\prime} 42^{\prime \prime}$ \\
1941003 & Baixo Guandu & $-19^{\circ} 31^{\prime} 25^{\prime \prime}$ & $-41^{\circ} 00^{\prime} 51^{\prime \prime}$ \\
1940012 & Itaguaçu & $-19^{\circ} 39^{\prime} 49^{\prime \prime}$ & $-40^{\circ} 50^{\prime} 07^{\prime \prime}$ \\
1940000 & Itarana & $-19^{\circ} 52^{\prime} 28^{\prime \prime}$ & $-40^{\circ} 52^{\prime} 28^{\prime \prime}$ \\
1841011 & Tumiritinga & $-18^{\circ} 58^{\prime} 35^{\prime \prime}$ & $-41^{\circ} 38^{\prime} 25^{\prime \prime}$ \\
1941005 & Conselheiro Pena & $-19^{\circ} 03^{\prime} 42^{\prime \prime}$ & $-41^{\circ} 31^{\prime} 58^{\prime \prime}$ \\
1941004 & Resplendor & $-19^{\circ} 20^{\prime} 35^{\prime \prime}$ & $-41^{\circ} 14^{\prime} 46^{\prime \prime}$ \\
1940006 & Colatina & $-19^{\circ} 31^{\prime} 51^{\prime \prime}$ & $-40^{\circ} 37^{\prime} 23^{\prime \prime}$ \\
\hline
\end{tabular}

transformada em uma variável dummy, apresentado a seguinte classificação: um (1), para vazões maiores ou iguais a de alerta de inundação $\left(4131 \mathrm{~m}^{3} / \mathrm{s}\right)$ e zero $(0)$ para valores abaixo das de alerta.

No modelo Logit utiliza-se uma função de distribuição acumulada logística, definida como:

$$
\begin{aligned}
\operatorname{Prob}\left(y_{t}=1\right) & =\frac{e^{\left(\beta_{0}+\sum_{i=1}^{k} \beta_{i} X_{t i}\right)}}{1+e^{\left(\beta_{0}+\sum_{i=1}^{k} \beta_{i} X_{t i}\right)}} \\
& =\frac{1}{1+e^{\left(\beta_{0}+\sum_{i=1}^{k} \beta_{i} X_{t i}\right)}} \times \frac{1}{e^{-\left(\beta_{0}+\sum_{i=1}^{k} \beta_{i} X_{t i}\right)}} \\
& =\frac{1}{1+e^{-\left(\beta_{0}+\sum_{i=1}^{k} \beta_{i} X_{t i}\right)}} \\
& =F\left(\beta X_{t}\right),
\end{aligned}
$$

em que, $F$ representa a função de distribuição logística; $X_{t i}$,variáveis independentes; $\beta=\left\{\beta_{0}, \beta_{1}, \cdots, \beta_{k}\right\}$, vetor de parâmetros a serem estimados; $k$, número de variáveis explicativas; $e$, base do logaritmo natural; e, $t=1, \cdots, n$ (número de observações). A variável $y_{t}$ representa uma dummy binária de uma determinada classificação de vazão, tal que $y_{t}=1$, se a vazão é maior ou igual a classificação de alerta para inundação e, $y_{t}=0$ se é menor do que a vazão de alerta para inundação. Da mesma forma, pode-se definir na Equação (1):

$$
\operatorname{Prob}\left(y_{t}=0\right)=\frac{1}{1+e^{\left(\beta_{0}+\sum_{i=1}^{k} \beta_{i} X_{t i}\right)}}=1-F\left(\beta X_{t}\right) .
$$

Segundo Silva et al. (2011), a esperança condicionada de $y_{t}$ é dada, portanto, por:

$$
\begin{aligned}
E\left(y_{t} / X_{t}\right) & =0 \times\left[\frac{1}{1+e^{\left(\beta_{0}+\sum_{i=1}^{k} \beta_{i} X_{t i}\right)}}\right] \\
& +1 \times\left(\frac{e^{\left(\beta_{0}+\sum_{i=1}^{k} \beta_{i} X_{t i}\right)}}{1+e^{\left(\beta_{0}+\sum_{i=1}^{k} \beta_{i} X_{t i}\right)}}\right) \\
& =\frac{e^{\left(\beta_{0}+\sum_{i=1}^{k} \beta_{i} X_{t i}\right)}}{1+e^{\left(\beta_{0}+\sum_{i=1}^{k} \beta_{i} X_{t i}\right)}} \\
& =\operatorname{Prob}\left(y_{t}=1\right) \\
& =F\left(\beta X_{t}\right) .
\end{aligned}
$$

Com o intuito de facilitar a estimação do vetor $(\beta)$ de parâmetros, o modelo logístico pode ser linearizado. Dividindo a Equação (1) pela Equação (2), obtém-se:

$$
\frac{\operatorname{Prob}\left(y_{t}=1\right)}{\operatorname{Prob}\left(y_{t}=0\right)}=\frac{F\left(\beta X_{t}\right)}{1-F\left(\beta X_{t}\right)}
$$




$$
\begin{aligned}
& =\frac{1+e^{\left(\beta_{0}+\sum_{i=1}^{k} \beta_{i} X_{t i}\right)}}{1+e^{-\left(\beta_{0}+\sum_{i=1}^{k} \beta_{i} X_{t i}\right)}} \\
& =e^{\left(\beta_{0}+\sum_{i=1}^{k} \beta_{i} X_{t i}\right)} .
\end{aligned}
$$

Aplicando o logaritmo natural na Equação (4) e denotando o resultado por Logit, , tem-se:

$$
\begin{aligned}
\operatorname{Logit}_{t}=\ln \left[\frac{F\left(\beta X_{t}\right)}{1-F\left(\beta X_{t}\right)}\right] & =\ln \left[e^{\left(\beta_{0}+\sum_{i=1}^{k} \beta_{i} X_{t i}\right)}\right] \\
& =\left(\beta_{0}+\sum_{i=1}^{k} \beta_{i} X_{t i}\right)
\end{aligned}
$$

Como os parâmetros da Equação (5) não são observáveis, segundo Gujarati e Porter (2008) para fins de estimação é considerado um componente de erro aleatório na Equação (5), assim esta equação pode ser reescrita como:

$$
\begin{aligned}
\text { Logit }_{t} & =\ln \left[\frac{F\left(\beta X_{t}\right)}{1-F\left(\beta X_{t}\right)}\right] \\
& =\left(\beta_{0}+\sum_{i=1}^{k} \beta_{i} X_{t i}+\epsilon_{t}\right),
\end{aligned}
$$

em que, $\epsilon_{t}$ é o erro aleatório. A Equação (6) é denominada de modelo Logit (Logistic probability unit), cujo termo foi proposto por Berkson (1944). Neste trabalho, a estimação do vetor de parâmetros ( $\beta$ ) foi feita pelo Método de Máxima Verossimilhança e a significância dos coeficientes foi analizada pelo teste z. Vale ressaltar que nesta pesquisa, quando o valor-p foi inferior a $10 \%$, as estatísticas foram consideradas significativas.

Para determinar o efeito marginal de cada variável preditora, sobre a probabilidade de ocorrência de vazão de alerta de inundação, é necessário usar os valores médios das variáveis explicativas. O efeito marginal da variável $X_{t i}$ sobre a variável dependente é descrito pela expressão:

$$
\frac{\partial y_{t}}{\partial X_{t i}}=\beta_{i} \frac{e^{-\left(\beta_{0}+\sum_{i=1}^{k} \beta_{i} X_{t i}\right)}}{\left[1+e^{-\left(\beta_{0}+\sum_{i=1}^{k} \beta_{i} X_{t i}\right)}\right]^{2}}
$$

A Equação (7) mostra o efeito marginal, sobre a probabilidade de $y_{t}=1$, de um aumento em $X_{t i}$. Para representar a variável explicativa $i\left(i \in \mathbb{N}^{*}\right)$ em questão, usou-se a notação matemática $X_{t i}$ e $\beta_{i}$ representa o parâmetro estimado desta variável. Observa-se que o efeito marginal de cada variável explicativa sobre a probabilidade não é constante, visto que depende do valor médio de cada variável $X_{t i}$. Para detalhes ver em Gujarati e Porter (2008), Hill et al. (2010), Silva et al. (2011), entre outros.

\section{Resultados e discussão}

Nas Tabelas 2 e 3 são apresentadas as estatísticas descritivas das variáveis vazão e precipitação. Os dados avaliados correspondem ao período de 01/01/1986 a 31/12/2014, perfazendo um total de 348 observações. Todas as análises foram feitas utilizando o software livre R (R Core Team, 2016). A presença de dados faltantes na série de vazões máximas mensais motivaram o uso da metodologia de imputação via algoritmo EM (expectation-maximisation), proposta por Junger e Leon (2015) e implementada na biblioteca R mtsdi (multivariante time-series data imputation).

Na Tabela 2 observa-se que, durante o período de estudo, a média das vazões máximas mensais foi de 1431,95 $\mathrm{m}^{3} / \mathrm{s}$, com desvio padrão de $1415,49 \mathrm{~m}^{3} / \mathrm{s}$ e coeficiente de variação de $98,85 \%$. Nesse contexto, tanto o desvio padrão quanto o coeficiente de variação resultaram em valores altos, permitindo concluir que a média é pouco representativa. De acordo com Bayer et al. (2012), a pouca representatividade supracitada pode ser decorrente da grande variabilidade intra-anual dos dados, ou seja, pela presença da sazonalidade. Nota-se, ainda, que os valores extremos observados, máximos e mínimos, foram de 9195,79 e 156,28 $\mathrm{m}^{3} / \mathrm{s}$, respectivamente. O primeiro foi registrado no dia 24/12/2013, enquanto o segundo foi observado no dia 07/09/2011. 
A distribuição da série de vazões máximas do rio Doce possui assimetria positiva igual a 2,19, isso significa que a média está do mesmo lado que a calda mais longa. Quanto a curtose, deve-se ressaltar que o valor encontrado foi igual a 5,91. Como a curtose caracteriza o grau de achatamento da distribuição, e a estimada foi superior a 3, a distribuição pode ser considerada leptocúrtica. Para detalhes consultar Fonseca e Martins (2011).

Tabela 2: Medidas descritivas da variável vazão

\begin{tabular}{lr}
\hline Estatísticas descritivas & Valor \\
\hline Média $\left(\mathrm{m}^{3} / \mathrm{s}\right)$ & 1431,95 \\
Mediana $\left(\mathrm{m}^{3} / \mathrm{s}\right)$ & 862,00 \\
Desvio padrão $\left(\mathrm{m}^{3} / \mathrm{s}\right)$ & 1415,49 \\
Coeficiente de variação \% & 98,85 \\
Valor máximo $\left(\mathrm{m}^{3} / \mathrm{s}\right)$ & 9195,79 \\
Valor mínimo $\left(\mathrm{m}^{3} / \mathrm{s}\right)$ & 156,28 \\
Assimetria & 2,19 \\
Curtose & 5,91 \\
\hline
\end{tabular}

A Tabela 3 apresenta as estatísticas descritivas para a precipitação máxima mensal medida em cada município considerado neste estudo. Em geral, observando-se os desvios padrão, o coeficiente de variação e as diferenças entre os máximos e mínimos, nota-se que a variável meteorológica apresenta grande variabilidade em termos estatísticos.

Tabela 3: Medidas descritivas da variável precipitação máxima mensal

\begin{tabular}{lrrrrrrrr}
\hline Estatísticas descritivas & \multicolumn{7}{c}{ Precipitação por Estações da ANA } \\
\cline { 2 - 8 } & TUMIR & CPENA & RESPL & AIMOR & ITAR & ITGUA & BGUAD & COLAT \\
\hline Média $(m m)$ & 27,64 & 26,34 & 28,09 & 29,14 & 30,41 & 31,33 & 25,31 & 29,98 \\
Mediana $(m m)$ & 19,10 & 19,90 & 21,30 & 22,60 & 22,80 & 20,35 & 19,80 & 24,55 \\
Desvio padrão $(m m)$ & 26,69 & 25,13 & 25,92 & 27,02 & 28,38 & 29,47 & 23,77 & 25,15 \\
Coeficiente de variação \% & 96,58 & 95,40 & 92,27 & 92,74 & 93,35 & 94,07 & 93,92 & 83,89 \\
Valor máximo $(m m)$ & 152,10 & 130,20 & 167,00 & 153,40 & 165,00 & 171,00 & 133,30 & 107,50 \\
Valor mínimo $(m m)$ & 0,00 & 0,00 & 0,00 & 0,00 & 0,00 & 0,00 & 0,00 & 0,00 \\
Assimetria & 1,40 & 1,11 & 1,38 & 1,34 & 1,56 & 1,31 & 1,41 & 1,04 \\
Curtose & 2,67 & 0,89 & 2,81 & 2,03 & 3,11 & 1,79 & 2,51 & 0,55 \\
\hline
\end{tabular}

A variação de grandes volumes de precipitação causa eventos extremos de vazão no rio Doce em Colatina, entre cheias e vazantes, o que resulta em enchentes em anos de intensa precipitação, como sucedeu em 1961, 1976, 1997, 2005, 2012 e 2013, e escassez hídrica, com pouca precipitação, como em 2014. De acordo com a análise dos dados da última inundação, de 19 e 24 de dezembro de 2013, feita por Dallapicola e Coelho (2015), a área urbana inundada pelo rio compreendeu cerca de $2,58 \mathrm{~km}^{2}$ da cidade de Colatina. Vale dizer que, a vazão atingida no evento extremo de 2013 foi de cerca de $9.028 \mathrm{~m}^{3} / \mathrm{s}$, sendo que o estimado para a maior vazão mensurável no município foi de 12.860 $\mathrm{m}^{3} / \mathrm{s}$, referente à enchente de fevereiro de 1979. Esses eventos a anos têm impactado a área urbana, provocando diversas perdas materiais e humanas. Além disso, esses acontecimentos alteraram a morfologia deste corredor fluvial, o que reforça a necessidade de um planejamento integrado que englobe o processo de ocupação urbana e os processos fluviais do rio Doce no contexto de uma importante bacia hidrográfica do país (Dallapicola e Coelho, 2015).

A Figura 2 apresenta a evolução temporal das vazões máximas mensais medidas no município de Colatina e a Figura 3 a da variável meteorológica no período de análise desse estudo. De um total de 348 observações, para a variável vazão, 21 representaram eventos de vazão de alerta de inundação. Como pode ser observada, Figura 2, a série apresenta um padrão intra-anual de variabilidade, com períodos de cheias seguidos por períodos de estiagem, o que caracteriza a propriedade de sazonalidade (Pinto et al., 2015).

As médias mensais plotadas dentro de seus meses formam as subséries (Figura 4), que confirmam a presença de sazonalidade e demonstram os períodos onde se concentram as vazões máximas e mínimas. Observa-se que o período de cheias é de outubro a março e o de seca de abril a setembro. Vale ressaltar que todos 21 eventos de vazões de alerta de inundação, ocorridos no período de tempo considerado, ocorreram na época de cheias. 


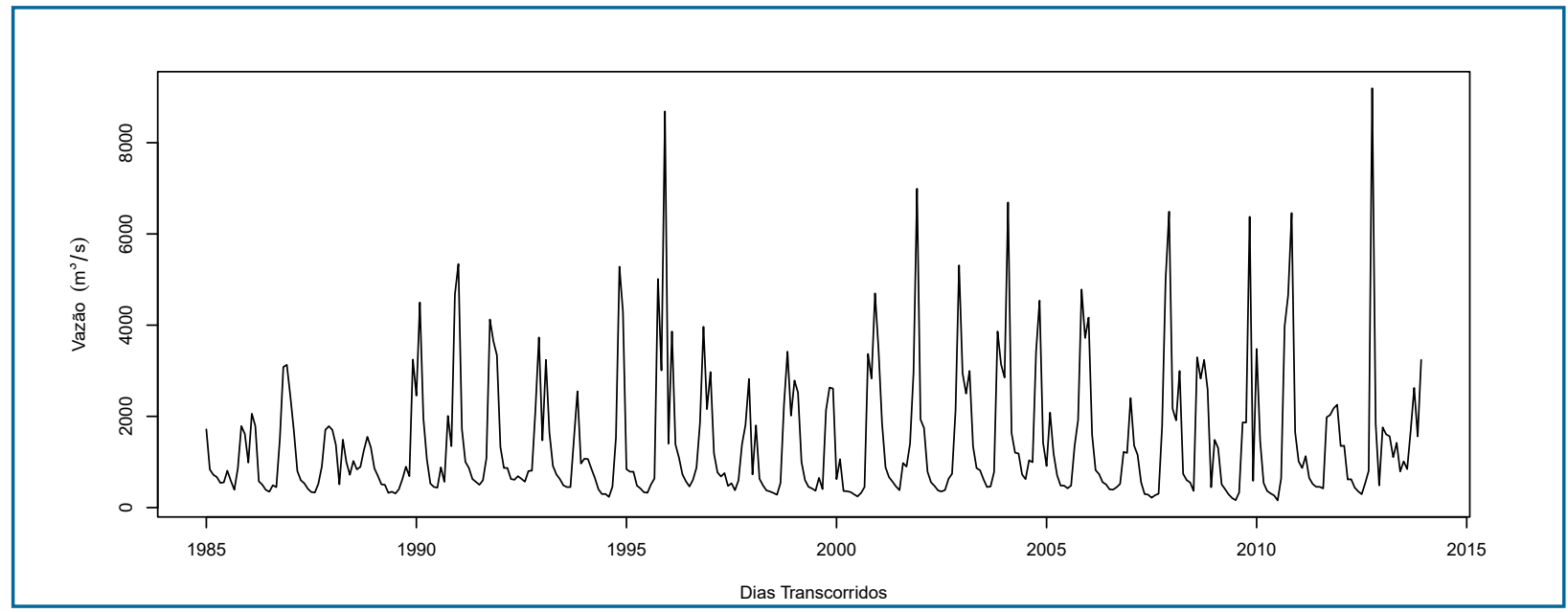

Figura 2: Vazões máximas mensais medidas no município de Colatina.

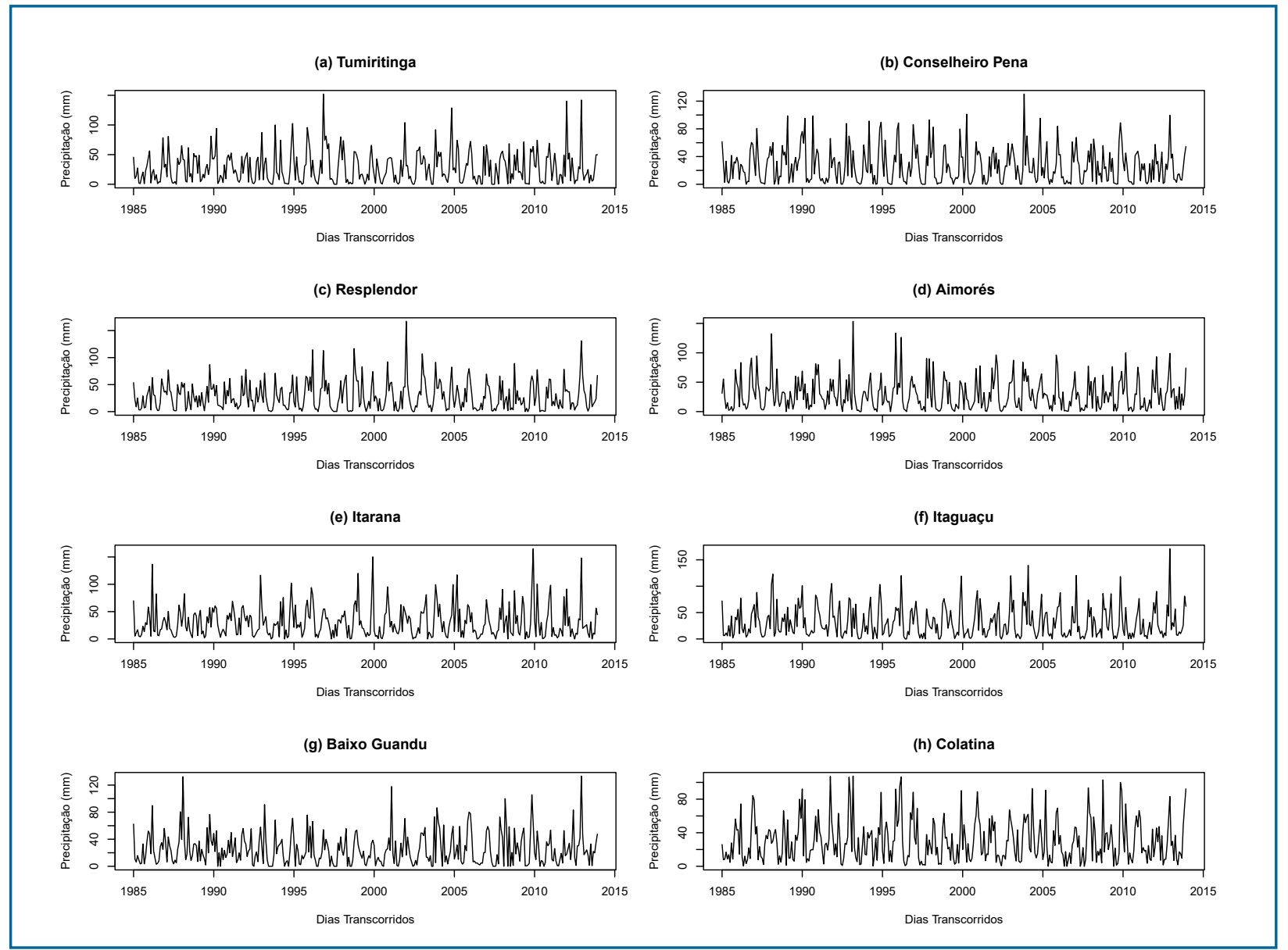

Figura 3: Precipitações máximas mensais para os municípios em estudo.

A Tabela 4 contém as correlações calculadas entre a série de vazão e as séries de precipitação de cada município considerado. Observa-se que a variável meteorológica apresenta relação linear positiva com vazões máximas do rio Doce em Colatina, conforme esperado. Segundo Júnior-Chierice e Landim (2014) a precipitação é a principal variável de informação de entrada de água na bacia hidrográfica e o escoamento se origina a partir delas, porém é influenciado por vários fatores tais como: topografia, porosidade, capacidade de infiltração e uso e ocupação do solo. Dessa forma, os eventos de vazões extremas estão diretamente relacionados com os eventos extremos de precipitação 


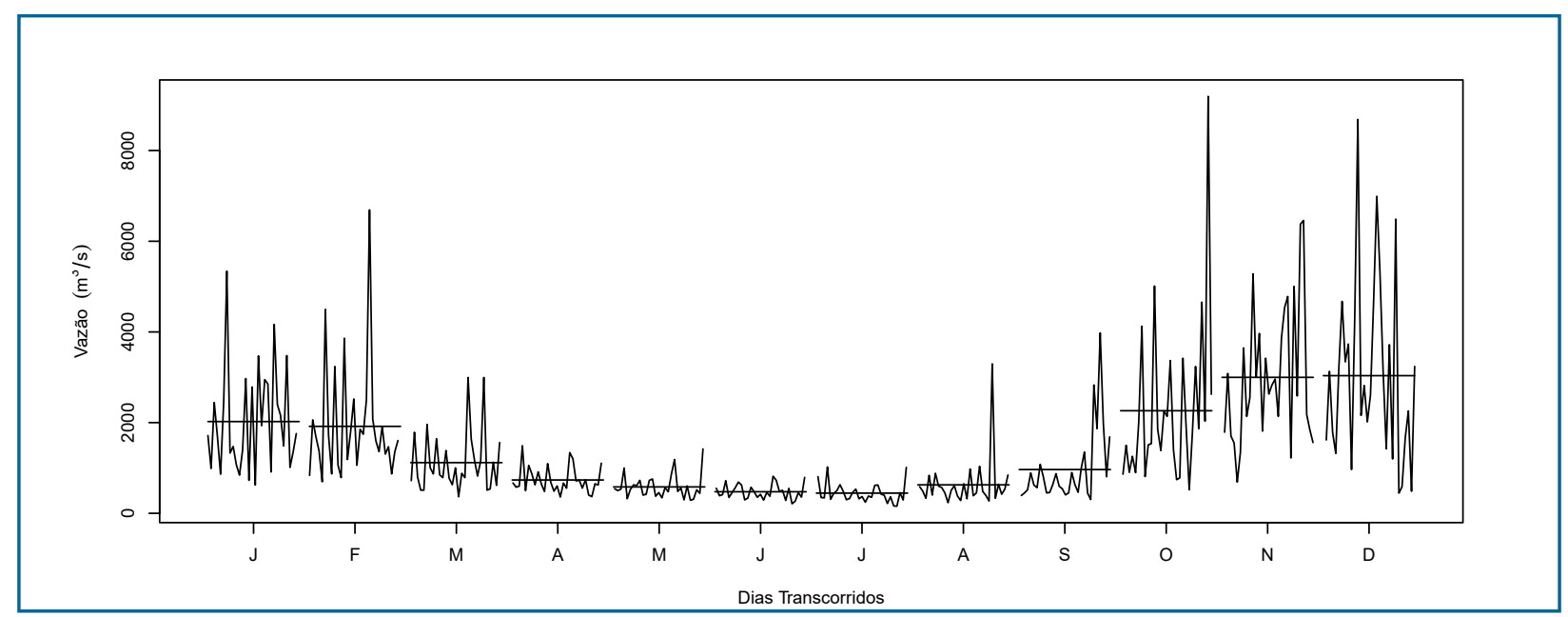

Figura 4: Subséries mensais da sazonalidade da série de vazões máximas mensais.

na bacia.

A Figura 5 apresenta a matriz de dispersão entre as vazões máximas mensais e a precipitação nos municípios de interesse. A Figura evidencia a associação entre as variáveis sob estudo. Os gráficos de dispersão corroboram com os resultados apresentados na Tabela 4.

Tabela 4: Matriz de correlação entre as variáveis sob estudo

\begin{tabular}{|c|c|c|c|c|c|c|c|c|c|}
\hline & Vazão & TUMIR & CPENA & RESPL & AIMOR & ITAR & ITGUA & BGUAD & COLAT \\
\hline Vazão & 1,00 & & & & & & & & \\
\hline TUMIR & 0,45 & 1,00 & & & & & & & \\
\hline CPENA & 0,45 & 0,76 & 1,00 & & & & & & \\
\hline RESPL & 0,43 & 0,67 & 0,64 & 1,00 & & & & & \\
\hline AIMOR & 0,37 & 0,56 & 0,60 & 0,71 & 1,00 & & & & \\
\hline ITAR & 0,40 & 0,56 & 0,55 & 0,63 & 0,60 & 1,00 & & & \\
\hline ITGUA & 0,44 & 0,55 & 0,58 & 0,67 & 0,65 & 0,70 & 1,00 & & \\
\hline BGUAD & 0,41 & 0,58 & 0,58 & 0,64 & 0,73 & 0,61 & 0,71 & 1,00 & \\
\hline COLAT & 0,50 & 0,54 & 0,58 & 0,64 & 0,63 & 0,65 & 0,68 & 0,63 & 1,00 \\
\hline
\end{tabular}

Na Tabela 5 são apresentados os resultados para a equação logística estimada para a série de vazões máximas mensais, considerando como variáveis explicativas a precipitação em Tumiritinga, Aimorés, Baixo Guandu e Colatina. Também são apresentados os respectivos efeitos marginais de cada variável sobre a probabilidade de ocorrência de vazões de alerta de inundação.

Observa-se que os coeficientes foram estatisticamente significativos de acordo com o teste $z$. Ressalta-se que, uma vez que a variável precipitação medida nos demais municípios contemplados neste estudo não foram estatisticamente significativas, as mesmas não foram incorporadas no ajuste do modelo final.

Usando a Equação (1), e mantendo-se a precipitação média constante nas estações de Tumiritinga, Baixo Guandu e Colatina, na condição de precipitação zero em Aimorés, tem-se 1,24\% de chance de ocorrer uma vazão de alerta em Colatina. Já para um dia com precipitação de $150 \mathrm{~mm}$ no referido município, tem-se 33,48\% de probabilidade de ocorrência de vazão de alerta de inundação.

Analisando os efeitos marginais (Tabela 5) verifica-se que, para cada unidade de acréscimo (um milímetro) na precipitação em Tumiritinga ou em Colatina, ocorreu um aumento de 0,04 pontos percentuais na probabilidade de ocorrer uma vazão de alerta de inundação em Colatina. Já uma elevação de um milímetro na precipitação em Aimorés ocasionou um aumento na probabilidade de vazão de alerta de 0,06 pontos percentuais. No mais, o aumento de uma unidade na precipitação no município de Baixo Guandu acarretou um aumento de 0,05 pontos percentuais na probabilidade de uma vazão de alerta em Colatina.

Analisando os efeitos marginais (Tabela 5) verifica-se que, para cada unidade de acréscimo (um milímetro) na 


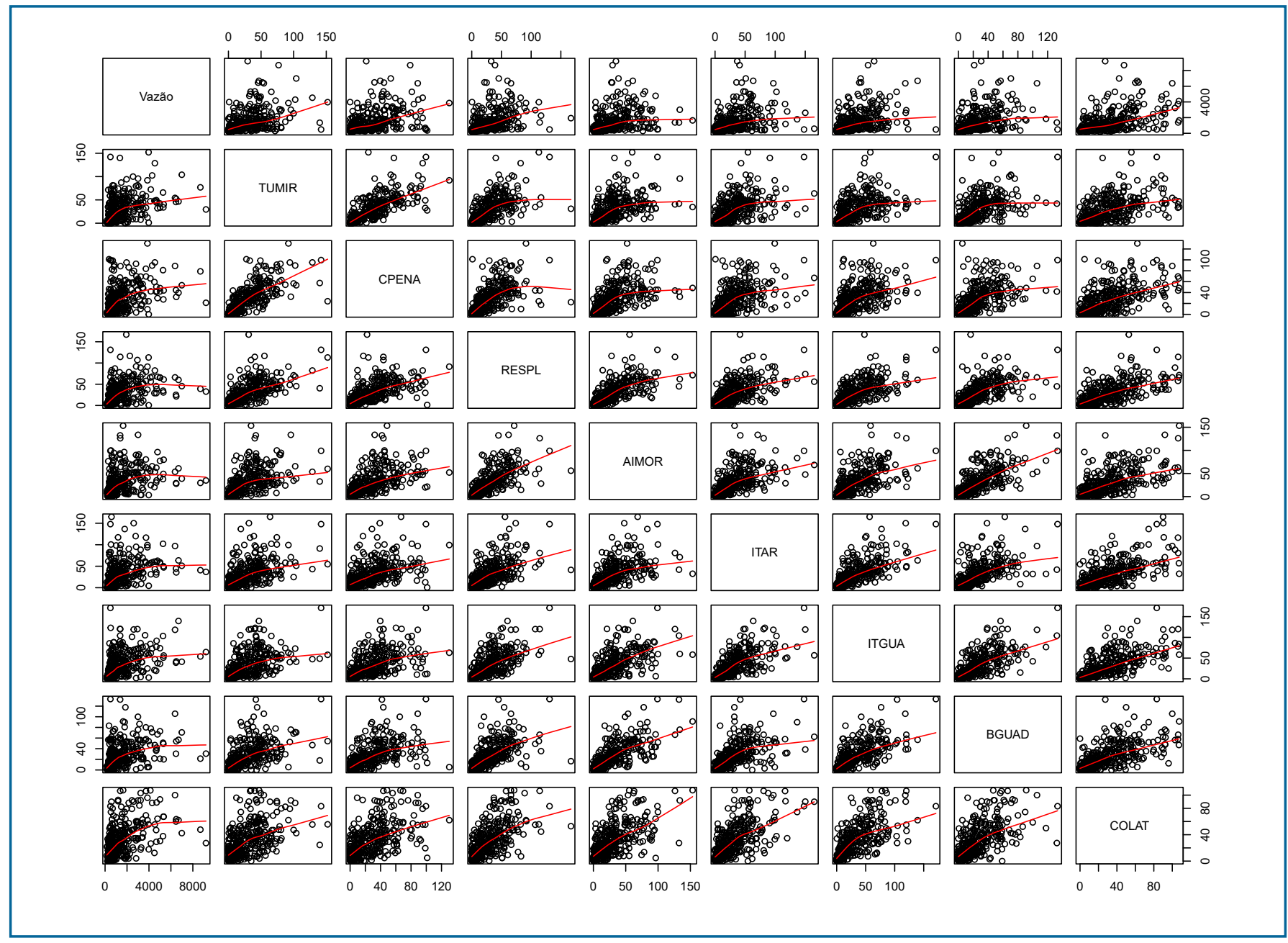

Figura 5: Correlação entre os valores de vazão observados em Colatina, ES, e precipitações observadas nos municípios em estudo.

precipitação em Tumiritinga ou em Colatina, ocorreu um aumento de 0,04 pontos percentuais na probabilidade de ocorrer uma vazão de alerta de inundação em Colatina. Já uma elevação de um milímetro na precipitação em Aimorés ocasionou um aumento na probabilidade de vazão de alerta de 0,06 pontos percentuais. No mais, o aumento de uma unidade na precipitação no município de Baixo Guandu acarretou um aumento de 0,05 pontos percentuais na probabilidade de uma vazão de alerta em Colatina.

Vale ressaltar que os sinais estimados são coerentes com o esperado. O sinal positivo dos coeficientes estimados das variáveis explicativas indicam a existência de uma relação direta entre as vazões máximas e essas variáveis, demonstrando que o aumento da precipitação nestes municípios eleva a vazão do rio Doce em Colatina, o que corrobora com os resultados encontrados na análise de correlação feita no presente estudo.

A fim de enriquecer o trabalho, também foram estimadas regressões logísticas para as estação do ano, primavera e verão (Tabela 6). Todos os 21 eventos extremos de vazões em Colatina ocorreram nestas duas estações. Como não ocorreram eventos extremos no inverno e no outono, não foi realizada a estimação de modelos para estas duas estações do ano.

De acordo com os resultados do teste $z$, apresentados na Tabela 6, os coeficientes estimados para as duas estações do ano, verão e primavera, foram estatisticamente significativos. Como era esperado, os coeficientes da primavera e do verão foram positivos, indicando que nos períodos com maiores volumes de chuva, a chance de ocorrência de um evento de vazão de alerta em Colatina aumentou. Analisando os efeitos marginais (Tabela 6) verifica-se que no verão a probabilidade de ocorrer um evento de vazão extrema elevou-se em 17,83 pontos percentuais. Já na primavera o efeito marginal igual a 0,0810 demonstra que nesta estação a probabilidade deste evento elevou-se em 8,1 pontos percentuais. 
Tabela 5: Equação logística considerando as variáveis explicativas e seus efeitos marginais

\begin{tabular}{lrrrrr}
\hline Variáveis & Coeficientes & Erro-padrão & Valor de Z & Valor-p & Efeito marginal \\
\hline Constante & $-5,8444^{*}$ & 0,6083 & $-9,6071$ & 0,0000 & \\
TUMIR & $0,0175^{* *}$ & 0,0059 & 2,9341 & 0,0033 & 0,0004 \\
AIMOR & $0,0246^{*}$ & 0,0061 & 4,0396 & 0,0001 & 0,0006 \\
BGUAD & $0,0184^{* *}$ & 0,0077 & 2,3737 & 0,0176 & 0,0005 \\
COLAT & $0,0173^{* *}$ & 0,0077 & 2,2337 & 0,0254 & 0,0004 \\
\hline
\end{tabular}

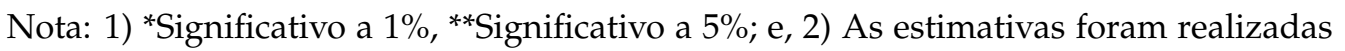
utilizando o método de covariância robusta GLM (Modelo Linear Generalizado).

Tabela 6: Equação logística considerando as variáveis explicativas (estações do ano) e seus efeitos marginais

\begin{tabular}{lrrrrr}
\hline Variáveis & Coeficientes & Erro-padrão & Valor de Z & Valor-p & Efeito marginal \\
\hline Constante & $-3,3322^{*}$ & 0,3392 & $-9,8227$ & 0,0000 & \\
Verão & $1,4996^{* *}$ & 0,4601 & 3,2589 & 0,0011 & 0,1783 \\
\hline Constante & $-3,0325^{*}$ & 0,2955 & $-10,2607$ & 0,0000 & \\
Primavera & $0,8730^{* * *}$ & 0,4596 & 1,8993 & 0,0575 & 0,0810 \\
\hline
\end{tabular}

Observações com variável dependente igual a zero: 327

Observações com variável dependente igual a um: 21

Total de observações $=348$

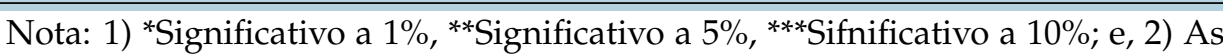
estimativas foram realizadas utilizando o método de covariância robusta GLM (Modelo Linear Generalizado).

Aplicando-se as informações de cada estação do ano na Equação (1), foi obtido que, no verão, a probabilidade de ocorrer um evento de vazão igual ou superior a de alerta é de 13,79\% e, na primavera, é de 10,34\%.

Os resultados apresentados neste trabalho corroboram os encontrados em Macêdo et al. (2013), os quais argumentam que o aumento da precipitação, assim como estações do ano com maior pluviosidade, proporcionam maiores chances de ocorrer um evento extremo de vazão.

Por fim, vale ressaltar que, o conhecimento da probabilidade de ocorrência de vazões máximas em corpos hídricos, que podem causar enchentes, é de extrema importância para adoção de medidas direcionadas ao controle das enchentes, sobretudo no meio urbano, dado que os prejuízos decorrentes dela transcendem aos prejuízos econômicos. De acordo com Nagem (2008), as águas pluviais após lavarem a bacia hidrográfica se misturam com a poluição difusa podendo ocasionar doenças como leptospirose, hepatites A e E, febre tifoide, entre outras. A autora ainda destaca que outros prejuízos podem ocorrer, a saber: danos a vias públicas; prejuízos aos veículos atingidos; aumento de acidentes; colapso nos serviços de infraestruturas; bem como perda de vidas humanas.

\section{Conclusões}

Este trabalho objetivou avaliar os impactos das precipitações máximas na bacia hidrográfica do rio Doce na probabilidade de ocorrência de episódios de vazão de alerta de inundação no município de Colatina, ES, Brasil, no período de 01/01/1986 a 31/12/2014, por meio do modelo Logit.

Os resultados do modelo de regressão logística demonstraram que existem impactos significativos das precipitações máximas dos municípios de Tumiritinga, Aimorés, Baixo Guandu e Colatina na probabilidade de ocorrência de episódios de vazão de alerta de inundação no município de Colatina, ES. Além disso, observou-se que nos períodos com maiores volumes de chuva (primavera e verão), a chance de ocorrência de um evento de vazão de alerta em 
Colatina aumentou significantemente.

Por fim, conclui-se que os resultados desse artigo podem subsidiar o planejamento municipal específico que auxilie na tomada de decisão para reduzir impactos, tais como: inundações em área urbana, em áreas de cultivo agrícola, danificações de estradas e sistemas de drenagem, que são causados pelos eventos extremos de precipitação.

\section{Referências}

Bayer, D. M., Castro, M. R., Bayer, F. M. (2012). Modelagem e previsão de vazões médias mensais do rio Potiribu utilizando modelos de séries temporais. Revista Brasileira de Recursos Hídricos, 17(2), 229-239.

Berkson, J. (1944). Aplication of the logistic function to bio-assay. Journal of the American statistical association, 39(227), 357-365.

Box, G., Jenkins, G. (1970). Time Series Analysis: Forecasting and Control, revised edn. San Francisco: Holden-Dayl.

Brito, F. A., Pinho, B. A. T. D. (2012). A dinâmica do processo de urbanização no Brasil, 1940-2010. Belo Horizonte: UFMG/CEDEPLAR.

C.B.H. Doce (2014). A bacia do rio Doce: Caracterização da bacia. Comitê da bacia hidrográfica do rio Doce, Governador Valadares, Minas Gerais, URL http://www.riodoce.cbh.gov.br/bacia_caractericao.asp.

Coelho, A. L. N. (2007). Alteração hidrogeomorfológica no médio-baixo rio Doce, ES. Tese (Doutorado em Geografia). Programa de Pós-Graduação em Geografia, Universidade Federal Fluminense. Niteroi, RJ.

CPRM - Serviço Geológico do Brasil (2015). Monitoramento Especial da Bacia do Rio Doce: Relatório I dezembro/2015. Belo Horizonte, Minas Gerais, URL http://www.cprm.gov .br/publique/media/hidrologia/eventos_ criticos/riodoce_relatoriol.pdf.

Dallapicola, M. S. Q., Coelho, A. L. N. (2015). Transbordamento das águas do rio doce na área urbana de Colatina-ES em dezembro de 2013. Anais XVII Simpósio Brasileiro de Sensoriamento Remoto - SBSR, João Pessoa-PB, Brasil, pp. 263-269.

Fonseca, J. S., Martins, G. A. (2011). Curso de Estatística, $6^{\circ}$ edn. Atlas.

Guha-Spir, D., Vos, F., Below, R., Ponserre, S. (2014). Annual Disater Statistical Review 2011. Brussel: CRED.

Gujarati, D. N., Porter, D. C. (2008). Basic Econometrics, $5^{\circ}$ edn. New York: McGraw-Hill/Irwin.

Hartmann, M., Moala, F. A., Mendonça, M. A. (2011). Estudo das precipitações máxima anuais em Presidente Prudente. Revista Brasileira de Meteorologia, 26(4), 561-568.

Hill, R. C., Judge, G. G., Griffiths, W. E. (2010). Econometria, $3^{\circ}$ edn. São Paulo: Saraiva.

Júnior-Chierice, N., Landim, P. B. (2014). Análise da chuva e vazão na bacia hidrográfica do rio Pardo. Geociências, $33(2), 244-260$.

Junger, W. L., Leon, A. P. (2015). Imputation of missing data in time series for air pollutants. Atmospheric Environment, 102, 96-104.

Koppen, W. (1900). Versuch einer klassifikation der klimate, vorzugsweise nach ihren beziehungen zur pflanzenweltl. Geogr Zeitschr, 6, 596-611.

Kuchenho, H., Thamerus, M. (1996). Extreme value analysis of munich air pollution data. Environmental and Ecological Statistics, 3(2), 127-141.

Macêdo, M. N. C., Dias, H. C. T., Coelho, F. M. G., Araújo, E. A., Souza, M. L. H., Silva, E. (2013). Precipitação pluviométrica e vazão da bacia hidrográfica do Riozinho do Rôla, Amazônia Ocidental. Revista Ambiente \& Água, $8(1), 206-221$.

Meller, A., Bravo, J. M., Collischonn, W. (2012). Análise de dados de vazão na previsão de cheias em tempo-real com o modelo hidrológico MGH-IPH. Revista Brasileira de Recursos Hídricos, 17(3), 209-224. 
Mendes, C. A. B., Vega, F. A. C. (2011). Técnicas de regressão logística aplicada à análise ambiental. Revista Geografia, 20(1), 5-30.

Mota, S. (2008). Gestão de Recursos Hídricos, $3^{\circ}$ edn. Rio de Janeiro: ABES.

Nagem, F. R. M. (2008). Avaliação econômicas dos prezuízos causados pelas cheias urbanas . 114 f. Dissertação (Mestrado em Ciências em Engenharia Civil). Programa de Pós-Graduação em Engenharia, COPPE, Universidade Federal do Rio de Janeiro. Rio de Janeiro, RJ.

Pinto, W. P., Lima, G. L., Zanetti, J. B. (2015). Análise comparativa de modelos de séries temporais para modelagem e previsão de regimes de vazões médias mensais do rio Doce, Colatina - Espírito Santo. Ciência e Natura, 37(4), 1-11.

R Core Team (2016). R: A Language and Environment for Statistical Computing. R Foundation for Statistical Computing, Vienna, Austria, URL https://www.R-project.org/.

Reis, J. B. C., Pons, N. A. D., Lopes, E. S. S. (2016). Monitoramento e alerta de inundação no município de Itajubá (MG) por regressão polinomial. Geociências, 35(1), 134-148.

Rodriguez, J., Guha-Spir, D., Vos, F., Below, R., Ponserre, S. (2009). Annual disaster statistical reviwe 2008: the numbers and trends. Belo Horizonte:UFMG/CEDEPLAR.

Silva, W. S., Paixão, A. N., Araújo, A. F. V., Picanço, A. P. (2011). Avaliação dos benefícios da coleta de lixo em Palmas, Tocantins: uma aplicação do método de avaliação contingentel. Engenharia Sanitária e Ambiental, 16(2), $141-148$.

Tucci, C. E. M. (2012). Hidrologia: ciência e aplicação, $4^{\circ}$ edn. Porto Alegre: UFRGS/ABRH.

Tucci, C. E. M., Porto, R. L. L., Barros, M. (2015). Drenagem urbana, $1^{\text {o }}$ edn. Porto Alegre: ABRH. 